\title{
Connaissances, attitudes et pratiques de la population du Hodh EI Gharbi (Mauritanie) face au virus de l'immunodéficience humaine(VIH).
}

\section{Awareness, attitudes, and practices of the population of the Hodh El Gharbi (Mauritania) against the human immunodeficiency virus (HIV).}

Boushab MB ${ }^{1}$, Fall-Malick FZ ${ }^{2,3}$, Diallo A ${ }^{4}$, Ould Salem ML ${ }^{3,5}$.

1- Service de Médecine Interne ; Centre Hospitalier d'Ä̈oun ; Mauritanie ;

2- Institut National d'Hépato-Virologie, Nouakchott ; Mauritanie ;

3- Faculté de Médecine de Nouakchott ; Mauritanie ;

4- Service de Médecine Polyvalente du Centre Hospitalier de Mayotte;

5- Laboratoire du Centre Hospitalier National de Nouakchott, Mauritanie.

Correspondance et tirés à part: Boushab Mohamed Boushab

Médecine interne ; Centre hospitalier d'Aïoun ; Hodh El Gharbi ; Mauritanie

\section{Résumé}

E-mail: bboushab@gmail.com

Il s'agit d'une enquête transversale, descriptive et analytique menée au centre hospitalier d'Aïoun sur la période du $1^{\mathrm{er}}$ janvier au 31 mai 2013. L'objectif global de notre travail était de décrire les connaissances, attitudes et pratiques sur le VIH/SIDA des patients reçus en consultation pour une infection sexuellement transmissible (IST). De façon spécifique, notre travail visait à évaluer les comportements sexuels à risque d'infection par le VIH et l'acceptation du test de dépistage volontaire chez ces consultants. Le dépistage volontaire du VIH n'était pas gratuit. L'information était recueillie à partir d'un questionnaire auto rempli. En cinq mois, 167 patients ont consenti à participer à l'étude soit 15,84\%.

Le sex-ratio H/F était de 0,65 et l'âge moyen était de $30 \pm 5$ ans [15-45ans]. Plus de la moitié $(58,1 \%)$ provenait du milieu rural. Parmi eux $89,2 \%$ étaient non instruits. Les connaissances générales sur les modes de transmission du VIH étaient erronées dans 94,6\% des cas. Le préservatif n'avait jamais été utilisé par les enquêtés $(91,6 \%)$. Le principal motif de consultation était les leucorrhées $(45,5 \%)$ suivies des mictions douloureuses $(22,2 \%)$. Le dépistage volontaire pour le VIH était effectué par 4,8\% des enquêtés. Un lien statistiquement significatif a été retrouvé entre la connaissance des modes de transmission du VIH, l'âge, le sexe, la provenance, le niveau d'éducation scolaire, le manque d'information et l'absence d'utilisation du préservatif.

Nos résultats ont révélé chez les consultants pour IST, des connaissances, attitudes et pratiques qui exposent à une grande vulnérabilité au VIH. A l'issue de ce travail, vu le lien étroit entre les IST et le VIH, nous recommandons le développement de stratégies globales de prévention (population générale) et ciblée (consultants IST) afin de réduire le risque d'infection par le VIH dans la région, en particulier chez les porteurs d'IST.

Mots clés : VIH, IST, fausses croyances, comportements, attitudes, Aïoun Summary

It is a cross-sectional study, descriptive and analytical, conducted in the Aïoun Hospital over a period from 1 January to 31 May 2013. Its main objective was to describe the knowledge, attitudes and practices toward HIV/AIDS in out-patients received for sexually transmitted infections (STI). The specific objectives of the study were to assess the sexual risk behaviors and the acceptance of voluntary HIV testing among STI consultants. HIV testing was not free. 
Information was collected from a questionnaire administered by an interviewer. In five months, 167 patients agreed to participate in the study was $15.84 \%$. The sex ratio $\mathrm{M} / \mathrm{F}$ was 0.65 and the average age was $30 \pm 5$ years [15-45years]. More than half $(58.1 \%)$ came from rural areas. Among them $89.2 \%$ were uneducated. General knowledge about modes of transmission of HIV / AIDS was incorrect in $94.6 \%$ of cases. The condom was never used by the respondents $(91.6 \%)$. The main reason for consultation was leucorrhea $(45.5 \%)$ followed by painful urination $(22.2 \%)$. Voluntary testing for HIV was performed in $4.8 \%$ by the respondents. A statistically significant link was found between knowledge of modes of HIV transmission, age, sex, origin, level of school education, lack of information and the non-use of condoms. Our results demonstrate the knowledge, attitudes and practices among consultants IST exposed to high vulnerability to HIV and show the efforts required to reduce vulnerability against STIs and HIV in rural areas.

Following this work, given the close link between STIs and HIV, we recommend the development of comprehensive prevention strategies (general population) and focused (IST consultants) to reduce the risk of HIV infection in the region, especially in carriers of STIs

Mots clés: HIV, STIs, false beliefs, behaviors, attitudes, Aïoun

\section{Introduction :}

Selon le rapport 2015 de l'ONUSIDA, 36,9 millions de personnes vivaient avec le VIH dans le monde à la fin de 2014 et les nombres les plus élevés de personnes vivant avec le VIH concernaient l'Afrique subsaharienne soit environ 24,7 millions de personnes [1]. En Mauritanie, le taux de prévalence chez les adultes de 15 à 49 ans est estimé à $0,6 \%$ [0,3\% - 0,9\%] en 2015 [2]. L'épidémie nationale est de type concentré avec une prévalence inférieure à $1 \%$ chez les femmes enceintes $(0,48$ en 2009 ) et des prévalences supérieures à $5 \%$, respectivement de 7,4\% et $9 \%$ en 2007 chez les professionnels du sexe (PS) et les consultants pour IST [3]. Malgré une prévalence nationale faible, les facteurs de propagation du virus sont multiples et les pratiques sexuelles à risque d'infection par le VIH semblent largement répandues au sein de la population générale et groupes plus vulnérables [3]. Les jeunes sont particulièrement vulnérables à l'infection à VIH en raison de leur comportement sexuel à risque d'infection par le VIH, de leur manque d'accès à l'information sur le VIH et aux services de prévention et l'insuffisance de leurs connaissances sur la transmission du VIH et les moyens de prévention [3,5]. En Mauritanie, une enquête sérologique chez les consultants IST remontant à l'année 1995 estimait la prévalence du VIH à $0,9 \%$ [4]..La première enquête seconde génération (socio-comportementale et sérologique) a été menée en 2008 chez le même groupe à Nouakchott et Nouadhibou, respectivement capitales politique et économique. Elle avait révélé des séroprévalences respectivement de $9 \%$ pour le VIH et de $10 \%$ pour la syphilis et que le préservatif était peu utilisé par les consultants, même lors des rapports occasionnels. L'enquête avait par ailleurs démontré que les consultants pour IST constituaient un groupe à risque d'infection par le VIH et la syphilis en raison de leurs comportements et de leur faible niveau de connaissance. En effet, 21,2\% des enquêtés avaient des partenaires en dehors de leur partenaire habituel et n'utilisaient pas le préservatif dans $75 \%$ des cas [5]. L'objectif principal de notre travail était de décrire les connaissances sur le VIH, les comportements, les attitudes et pratiques des patients reçus en consultation pour une infection sexuellement transmissible (IST) 
dans le Centre Hospitalier Régional d'Aïoun en Mauritanie, situé au niveau régional.

\section{Cadre de l'étude}

La présente étude qui s'est déroulée au Centre Hospitalier Régional d'Aïoun (région du Hodh El Gharbi), ville du SudEst de la Mauritanie située à $800 \mathrm{~km}$ de Nouakchott (capitale). La population du Hodh El Gharbi est estimée à 249768 habitants et celle d'Aïoun à 11867 habitants [8]. Les principales activités de leurs populations sont l'élevage et le commerce. Le Centre Hospitalier d'Aïoun assure les services d'un hôpital du jour pour la population environnante et les services de référence pour toute la région.

\section{Patients et méthodes}

Il s'agissait d'une enquête transversale, descriptive et analytique menée au centre hospitalier régional d'Aïoun durant une période allant $\mathrm{du} 1^{\mathrm{er}}$ janvier au 31 mai 2013. Ont été inclus tous les patients consultant pour un écoulement urétral, une ulcération génitale ou tout autre symptôme d'IST, d'âge compris entre 15 et 45 ans et qui avait donné son consentement oral pour participer à l'enquête. Ont été exclus, les patients d'âge inferieur à 15 ans et supérieur à 45 ans et les patients répondant aux critères d'inclusion mais ne voulant pas participer à l'enquête. Le diagnostic clinique et la prise en charge des IST étaient basés sur l'approche syndromique étant donné qu'il n'existe pas de laboratoire de Microbiologie à l'hôpital. Les patients présentant une IST étaient à leur charge en ambulatoire. Le recueil des informations était fait à l'aide d'une fiche d'entretien individuelle renseignée par un enquêteur après avoir expliqué l'intérêt de l'enquête et obtenu le consentement de l'enquêté. Le consentement oral était libre et éclairé. Les informations ont porté sur les caractéristiques sociodémographiques, les connaissances, attitudes et pratiques vis-à-vis du VIH. Le diagnostic de l'infection à VIH a été retenu devant la positivité du test rapide (DetermineTM ${ }^{\circledR}$ HIV-1/2 (Alere Medical Co. Ltd, Japan). La saisie des données et l'analyse statistique ont été réalisées à l'aide du logiciel Epi Info 3.5.3 (CDC Atlanta USA). Le test de Khi 2 et l'intervalle de confiance (I.C) à 95\% ont été appliqués pour établir une comparaison entre différentes proportions, considérée significative pour un $\mathrm{p}<0,05$. Le logiciel Excel a été utilisé pour la production des graphiques descriptifs relatifs aux croisements de variables et différents indicateurs.

\section{Résultats}

Durant notre période d'enquête, 237 consultants avaient présenté une IST. Parmi eux, 167 soit 70,4\% ont consenti à participer à l'étude. Le sex-ratio Homme/Femme est de 0,65. Parmi les enquêtés $53,9 \%$ avaient moins de 30 ans. La majorité $(89,2 \%)$ des enquêtés n'était pas scolarisée ou n'a pas dépassé le niveau primaire. Plus de la moitié des enquêtés $(58,1 \%)$ provenait du milieu rural c'est-àdire hors de la ville d'Aïoun. Les connaissances erronées sur les modes de transmission de l'infection étaient fréquentes dans $94,6 \%$ des cas. En effet, les fausses croyances telles que les piqûres des moustiques, et côtoyer une personne vivant avec le VIH (PVVIH) étaient considérées comme moyen de transmission de l'infection par respectivement $37,2 \%$ et $19,8 \%$ des enquêtés. Les sources d'information et d'acquisition de connaissances (télévision, radio et journaux) ne sont citées que dans 13,8\% des cas. L'évaluation des comportements sexuels a montré que $91,6 \%$ des consultants avaient des rapports sexuels non protégés avec des partenaires multiples durant les 12 derniers mois et n'avaient jamais utilisé de préservatifs (Tableau I). Une proportion de $4,8 \%$ a accepté de faire 
le test de dépistage volontaire pour le VIH. La sérologie du VIH était positive dans la moitié des cas. Globalement les principaux motifs de recours à la consultation étaient regroupés dans la figure 1. Un lien statistiquement significatif était retrouvé entre les connaissances des modes de transmission et l'âge, le sexe, la provenance, le niveau d'instruction, le manque d'information, la non utilisation du préservatif et les rapports sexuels non protégés (Tableau II).

\section{Discussion}

Durant notre enquête, $70,4 \%$ consultants pour IST ont accepté de participer à l'étude. Ces consultants pour IST constituent un groupe à risque vis-à-vis de l'infection à VIH en Mauritanie en raison de leurs comportements et de leur bas niveau de connaissance associés à une prévalence élevée de l'ordre de $9 \%$ comme l'avait souligné Kebé et al. dans une étude menée à Nouakchott et Nouadhibou [5]. L'âge moyen des enquêtés était de $30 \pm 5$ ans avec une prédominance féminine (60,5\%). La majorité $(89,2 \%)$ des enquêtés n'était pas scolarisée ou n'a pas dépassé le niveau primaire. Plus de la moitié de nos enquêtés $(58,1 \%)$ provenait du milieu rural de la Wilaya (région) du Hodh Gharbi, voisin de la capitale, Aïoun. Cette prédominance était probablement liée au choix du Centre Hospitalier d'Aïoun (capitale régionale), centre de référence de la région, fournissant des soins de qualité en comparaison avec les postes de santé implantés au niveau rural. Les connaissances générales des consultants enquêtés sur l'infection par le VIH étaient erronées, notamment en ce qui concerne les modes de transmission et les moyens de prévention. Ainsi, la majorité $(94,6 \%)$ des enquêtés ne savait pas que l'infection par le VIH est une pathologie grave et que les rapports sexuels non protégés constituaient un principal mode de transmission. Ce bas niveau de connaissances générales sur l'infection par le VIH constaté au niveau de notre population d'étude pourrait être lié d'une part au bas niveau d'instruction, empêcherait les enquêtés d'accéder à l'information et d'autre part par le fait que les sujets relatifs à la sexualité demeurent tabous et donc très difficilement évoqués. Dans notre étude, les fausses croyances telles que les piqûres des moustiques $(37,1 \%)$ et côtoyer une personne vivant avec le VIH (19,8\%) étaient incriminées dans les voies de transmission de l'infection. Par ailleurs, seulement 13,8\% des enquêtés avaient déjà entendu parler du VIH/SIDA. Le rôle important des médias (télévision, radio et journaux) comme source d'information dans l'acquisition des connaissances, était noté dans plusieurs études [5, 7]. L'évaluation des comportements sexuels a montré que $91,6 \%$ des enquêtés avaient des rapports sexuels non protégés avec des partenaires occasionnels durant les 12 derniers mois qui précédaient la consultation et n'avaient jamais utilisé le préservatif. La multiplicité des partenaires sexuels et l'utilisation irrégulière des préservatifs étaient rapportées par d'autres auteurs [5, 9]. Le faible taux d'utilisation des préservatifs que nous avons retrouvé serait essentiellement lié à sa méconnaissance. Cette situation, pourrait s'expliquer part le déficit notable d'activités de prévention sur l'infection par le VIH et les autres IST au sein de la population associé à des conditions favorables à des pratiques sexuelles à risque d'infection par le VIH, notamment, la précocité des premiers rapports sexuels, le multipartenariat sexuel [5-8] et l' exposition à des grossesses non planifiées [9-14]. Parmi les enquêtés, seulement $4,8 \%$ ont affirmé avoir fait leur test de dépistage pour connaître leur statut sérologique. Cette proportion est largement inferieure à celle observée par Touré et al [9]. Ce faible taux de dépistage pourrait s'expliquer d'une part, à la non gratuité du test au niveau du Centre 
Hospitalier et d'autre part, par le fait qu'il n'était demandé que dans un but diagnostique.

Le mode de recueil des données collectées a pu constituer une limite pour notre étude. En effet l'interview était effectuée en langues nationales pour permettre aux consultants de mieux comprendre les questions. Malgré cette mesure, les interprétations des enquêtés ont pu entraîner des incompréhensions, voire des modifications du sens d'une question posée. Il est évident que les réponses aux questions sur le vécu du VIH/SIDA, sujet sensible, n'ont probablement pas toujours été franches, même si l'anonymat des informations recueillies était garanti.

Dans notre étude, le principal motif de consultation était les leucorrhées $(45,5 \%)$, suivi des mictions douloureuses $(22,2 \%)$, alors que, Kebe et al [5], avaient observé comme principaux motifs de consultation, dans une étude similaire menée dans le pays en 2008, les brulures mictionnelles $(34,45 \%)$ et les leucorrhées $(28,89 \%)$. Dans notre étude, il a été démontré un lien statistiquement significatif entre l'âge, le sexe, la provenance, le niveau d'instruction, la sous information médiatique, l'utilisation irrégulière $\mathrm{du}$ préservatif, les pratiques sexuelles à risque et les déterminants des connaissances sur l'infection par le VIH. Les jeunes (1530ans) constituant $53,9 \%$ de notre échantillon d'étude sont particulièrement vulnérables à l'infection à VIH en raison de leur grande activité sexuelle associé à des comportements à risque d'infection par le VIH et de leur manque d'accès à l'information sur cette infection (modes de transmission et moyens de prévention) $[8$, $15]$.

\section{Conclusion}

Notre étude menée au Centre Hospitalier d'Aïoun a porté sur des patients consultants pour IST. Elle a révélé des connaissances, comportements, attitudes et pratiques à risque d'infection par le $\mathrm{VIH}$, associés à un faible recours au test de dépistage du VIH.

$\mathrm{Au}$ terme de cette étude, nous recommandons d'instaurer des activités continues de sensibilisation au profit de la population de la région, en particulier celui du milieu rural qui a peu d'accès à l'information; et de mener des activités régulières de sensibilisation ciblées vers les consultants pour IST au niveau des structures de santé.

Pour être efficaces, ces interventions doivent impliquer activement les leaders communautaires, les religieux et organisations de la société civile (ONG, coopératives...) et associer les medias, en particulier la radio (canal de communication le plus accessible au niveau régional).

\section{Conflit d'intérêt}

Pas de conflit d'intérêt.

\section{Bibliographie :}

1. Rapport

ONU/SIDA

2015.http://www.unaids.org/sites/default/files/media_asset/AIDS_by_the_numbers_2015_fr.p df (consulté, le 15/08/2016).

2. http://www.unaids.org/fr/regionscountries/countries/mauritania/ (consulté, le 15/08/2016).

3. Rapport d'activités sur la réponse au SIDA en Mauritanie (GARPR), 2014. www.unaids.org/fr/regionscountries/countries/mauritania/ (consulté, le 15/08/2016). 
4. Lo B, Philippon M, Cunin P, Meynard D, Diagana MT. Etiologie microbienne des écoulements génitaux à Nouakchott, Mauritanie.Bull Soc Pathol Exot 1997;90(2):81-2.

5. Kebé M, Fall-Malick FZ, Eghady OIet al..Séroprévalence VIH chez les consultants IST à Nouakchott et à Nouadhibou (Mauritanie). Bull Soc Pathol Exot 2011;104:374-7.

6. Konan Y, Angbo-Effi O, Koffi M et al. Comportements sexuels des adolescents en Côte d'Ivoire. Cahiers de Sante Pub 2003;2:37-44.

7. Institut National de Recherches en Santé Publique. Enquêtes sérologiques et comportementales sur le VIH/SIDA et les IST chez les camionneurs, marins et pêcheurs en 2007 : rapport de synthèse. Ministère de la Santé de Mauritanie (2009).

8. Office National de la Statistique et pour ORC MACRO. Enquête démographique et de santé, Mauritanie 2000-2001. Calverton, Maryland, USA:ONS et ORC Macro (2001).

9. Touré B, Koffi K, Kouassi-Gohou V et al.. Connaissances, attitudes et pratiques des collégiens et lycéens d'Abidjan face au VIH/sida. Med Trop 2005;65:346-8

10. Rotermann M. Relations sexuelles, condoms et MTS chez les jeunes. Rapports sur la santé 2005;16(3):47-53.

11. Sellors JW, Mahony JB, Kaczorowski J et al. Prevalence and predictors of human papillomavirus infection in women in Ontario, Canada. CM AJ 2000;163(5):503-8.

12. Heck JE, Berthiller J, Vaccarella $S$ et al. Sexual behaviors and the risk of head and neck cancers: a pooled analysis in the International Head and Neck Cancer Epidemiology (INHANCE) consortium: Int J Epidemiol 2010;39:166-81.

13. Wellings K, Nanchanhal K, Macdowall W et al. Sexual behavior in Britain: early heterosexual experience: Lancet 2001;358:1843-50.

14. Abma JC, Martinez GM, Copen CE et al. Teenagers in the United States: Sexual activity, contraceptive use, and childbearing, National Survey of Family Growth 2006-2008.Vital Health Stat 2010;23(30):1-57.

15. Gilbert M, Li X, Petric M et al. Using centralized laboratory data to monitor trends in Herpes Simplex Virus type 1 and 2 infections in British Columbia and the changing etiology of genital herpes. Can J Public Health, 2011;102(3):225-9. 
Tableau I : Caractéristiques sociodémographiques, évaluation des connaissances des consultants sur les modes de transmission et le dépistage de l'infection VIH.

Table I: Demographics data of outpatients and assessment of their knowledge about the transmission and the screening of HIV infection.

\begin{tabular}{llll}
\hline Paramètres & & Effectif & Pourcentage \\
\hline Age & $15-30$ & 90 & 54,9 \\
Sexe & $31-45$ & 77 & 46,1 \\
\multirow{2}{*}{ Provenance } & Féminin & 101 & 60,5 \\
& Masculin & 66 & 39,5 \\
Niveau d'instruction & Sub urbaine & 97 & 58,1 \\
& Urbaine & 70 & 41,9 \\
Situation matrimoniale & Primaire ou aucun & 149 & 89,2 \\
& Secondaire ou supérieur & 18 & 10,8 \\
& Mariés & 91 & 54,5 \\
Evaluation des connaissances & Célibataires & 39 & 23,4 \\
\multirow{2}{*}{ Piqure de moustique } & Veufs/divorcés & 37 & 22,1 \\
& Erronées & 158 & 94,6 \\
Côtoyer un PVVIH* & Bonnes & 9 & 5,4 \\
& Non & 105 & 62,9 \\
Les sources d'informations & Oui & 62 & 37,1 \\
& Non & 134 & 80,2 \\
Utilisation du préservatif & Oui & 33 & 19,8 \\
& Non & 144 & 86,2 \\
Dépistage VIH & Oui & 23 & 13,8 \\
& Non & 153 & 91,6 \\
& Oui & 14 & 8,4 \\
& Non & 159 & 95,2 \\
& Oui & 8 & 4,8 \\
\hline
\end{tabular}

PVVIH* : Personne vivant avec le VIH 


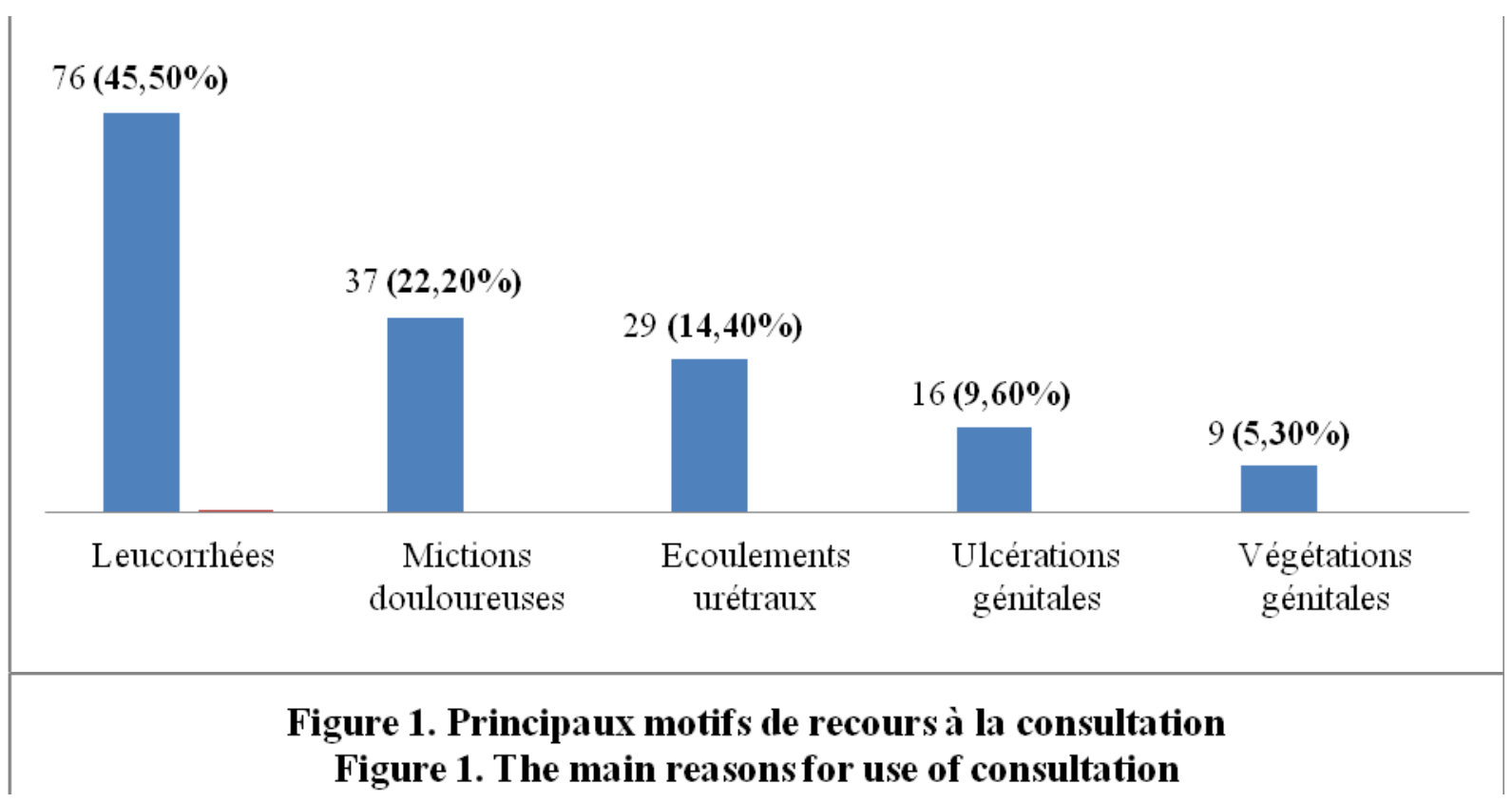

Tableau II : Les déterminants des connaissances.

Table II: Determinants of knowledge. 
[Article Original Connaissances, attitudes et pratiques face au VIH]

\begin{tabular}{|c|c|c|c|c|}
\hline \multicolumn{2}{|l|}{ Paramètres } & \multicolumn{2}{|c|}{ Appréciation des connaissances } & \multirow[t]{2}{*}{$\mathbf{P}$} \\
\hline & & Bonnes & Erronées & \\
\hline \multicolumn{5}{|l|}{ Age } \\
\hline & $15-30$ & 0 & $90(100)$ & 0,0007 \\
\hline & $31-45$ & $9(11,7)$ & $68(88,3)$ & \\
\hline \multicolumn{5}{|l|}{ Sexe } \\
\hline & Féminin & $1(1)$ & $100(99)$ & 0,002 \\
\hline & Masculin & $8(12,1)$ & $58(87,9)$ & \\
\hline \multicolumn{5}{|l|}{ Provenance } \\
\hline & Rurale & 0 & $97(100)$ & 0,0002 \\
\hline & Urbaine & $9(12,9)$ & $61(87,1)$ & \\
\hline \multicolumn{5}{|l|}{$\begin{array}{l}\text { Niveau } \\
\text { d'étude }\end{array}$} \\
\hline & Aucun ou primaire & 0 & $149(100)$ & $10^{-9}$ \\
\hline & Secondaire ou plus & $9(50)$ & $9(50)$ & \\
\hline \multicolumn{5}{|l|}{$\begin{array}{l}\text { Connaissance } \\
\text { d'une source } \\
\text { d'informations }\end{array}$} \\
\hline & Oui & $9(39,1)$ & $14(60,9)$ & $10^{-8}$ \\
\hline & Non & 0 & $144(100)$ & \\
\hline \multicolumn{5}{|c|}{ Utilisation du préservatif } \\
\hline & Oui & $6(42,9)$ & $8(57,1)$ & 0,000008 \\
\hline & Non & $3(2)$ & $150(98)$ & \\
\hline
\end{tabular}

